\title{
Banks-Casher-type relations for complex Dirac spectra*
}

\author{
Takuya Kanazawa \\ Quantum Hadron Physics Laboratory, RIKEN, Saitama 351-0198, Japan \\ E-mail: takuya.kanazawaeriken.jp \\ Tilo Wettig ${ }^{\dagger}$ \\ Department of Physics, University of Regensburg, 93040 Regensburg, Germany \\ E-mail: tilo.wettig@ur.de
}

\section{Naoki Yamamoto}

Department of Physics, University of Maryland, College Park, MD 20742-4111, USA

E-mail: nyama@umd. edu

For theories with a sign problem there is no analog of the Banks-Casher relation. This is true in particular for QCD at nonzero quark chemical potential. However, for QCD-like theories without a sign problem the Banks-Casher relation can be extended to the case of complex Dirac eigenvalues. We derive such extensions for the zero-temperature, high-density limits of two-color QCD, QCD at nonzero isospin chemical potential, and adjoint QCD. In all three cases the density of the complex Dirac eigenvalues at the origin is proportional to the BCS gap squared.

31st International Symposium on Lattice Field Theory LATTICE 2013

July 29 - August 3, 2013

Mainz, Germany

* Supported by RIKEN iTHES Project and JSPS KAKENHI Grants Number 25887014 (TK), by DFG (TW), and by Maryland Center for Fundamental Physics (NY).

† Speaker. 


\section{Introduction}

The Banks-Casher relation $|\langle\bar{\psi} \psi\rangle|=\pi \rho(0)[1]$, where $\langle\bar{\psi} \psi\rangle$ is the chiral condensate and $\rho(\lambda)$ is the Dirac eigenvalue density, links the order parameter for spontaneous chiral symmetry breaking to an accumulation of Dirac eigenvalues near zero. Specifically, chiral symmetry breaking occurs if the low-lying Dirac eigenvalues are of order $1 / V_{4}$, where $V_{4}$ is the space-time volume. For the derivation of the Banks-Casher relation it is essential that the fermionic measure is positive definite [2]. If it is not, as is the case in QCD at nonzero density, $\rho(0)$ is undefined and the connection between $\langle\bar{\psi} \psi\rangle$ and the (complex) Dirac spectrum is more complicated [3, 4]. Here, we consider QCD-like theories at high density (and zero temperature) that have complex Dirac spectra but positive definite fermionic measures. We show that Banks-Casher-type relations can be obtained for these theories, which in all cases have the form

$$
\Delta^{2}=\frac{2 \pi^{3}}{3 d_{\text {rep }}} \rho(0),
$$

where $\Delta$ is the BCS gap, $\rho(\lambda)$ is now a two-dimensional spectral density, and $d_{\text {rep }}$ is the dimension of the color group representation in which the fermions transform. To derive these relations, we had to construct the corresponding low-energy effective theories. All details can be found in [5].

\section{Derivation of Banks-Casher-type relations}

\subsection{Preliminaries}

The main idea of the derivation is to write down the partition function $Z(M)$ as a function of the quark mass matrix $M$, both in the fundamental (QCD-like) theory and in the corresponding effective theory. Taking suitable derivatives then yields $\sim \rho(0)$ in the fundamental theory and $\sim \Delta^{2}$ in the effective theory. The Banks-Casher-type relation is obtained by identifying these results.

Obviously, the effective theory is needed for this derivation. For two-color QCD it was constructed previously [6], while for the isospin and adjoint cases it was constructed in [7, 5]. Several points are worth noting in this respect: (a) At high density the explicit breaking of $\mathrm{U}(1)_{A}$ is strongly suppressed because instantons are screened. Instead, $\mathrm{U}(1)_{A}$ is broken spontaneously by the diquark condensate. (b) For chemical potential $\mu \gg \Lambda_{\mathrm{QCD}}$ the diquark pairing is of the BCS type since there is an attractive channel between quarks near the Fermi surface. (c) Because the fermionic measure is positive definite, QCD inequalities allow us to exclude certain symmetry-breaking patterns. (d) The coefficient of the mass term in the effective theory is not a free parameter but can be computed in high-density effective theory (HDET) [8].

\subsection{Two-color QCD}

For simplicity we start with $N_{f}=2$ flavors. The partition function of two-color QCD is then

$$
Z^{\left(N_{f}=2\right)}=\left\langle\operatorname{det}\left(D+m_{1}\right) \operatorname{det}\left(D+m_{2}\right)\right\rangle_{\mathrm{YM}}=\left\langle\operatorname{det}\left(D+m_{1}\right) \operatorname{det}\left(D^{\dagger}+m_{2}\right)\right\rangle_{\mathrm{YM}},
$$

where $D=D(\mu)=\gamma_{v} D_{v}+\mu \gamma_{4}$ is the Euclidean Dirac operator, the average is with respect to the Yang-Mills action, and in the last step we have used the anti-unitary symmetry $\left[C \gamma_{5} \tau_{2} K, D\right]=0$ 
that is specific to gauge group $\mathrm{SU}(2)$. (In this relation, $C$ is the charge conjugation operator, $\tau_{2}$ is the second generator of the gauge group, and $K$ is the complex conjugation operator.) To have a positive-definite measure we could either choose $m_{1}=m_{2}=m$, which leads to some mathematical subtleties [5], or $m_{1}=z$ and $m_{2}=z^{*}$ with $z \in \mathbb{C}$. We make the latter choice. In terms of the Dirac eigenvalues we then have

$$
Z^{\left(N_{f}=2\right)}=\left\langle\prod_{n}\left[\left(\lambda_{n}+z\right)\left(\lambda_{n}^{*}+z^{*}\right)+\varepsilon^{2}\right]\right\rangle_{\mathrm{YM}},
$$

where we have introduced a regulator $\varepsilon$ for later use. Taking derivatives first and the thermodynamic and chiral limits (in this order) thereafter yields

$$
\lim _{z \rightarrow 0} \lim _{V_{4} \rightarrow \infty} \frac{1}{V_{4}} \frac{\partial^{2} \log Z}{\partial z \partial z^{*}}=\int d^{2} \lambda \frac{\varepsilon^{2}}{\left(|\lambda|^{2}+\varepsilon^{2}\right)^{2}} \rho(\lambda)+\int d^{2} \lambda \int d^{2} \lambda^{\prime} \frac{\lambda^{*}}{|\lambda|^{2}+\varepsilon^{2}} \frac{\lambda^{\prime}}{\left|\lambda^{\prime}\right|^{2}+\varepsilon^{2}} \rho_{2}^{c}\left(\lambda, \lambda^{\prime}\right)
$$

with spectral density and connected two-point function defined by

$$
\begin{aligned}
\rho(\lambda) & =\lim _{z \rightarrow 0 V_{4} \rightarrow \infty} \lim _{V_{4}}\left\langle\frac{1}{n}\left\langle\delta^{2}\left(\lambda-\lambda_{n}\right)\right\rangle_{N_{f}=2},\right. \\
\rho_{2}^{c}\left(\lambda, \lambda^{\prime}\right) & =\lim _{z \rightarrow 0 V_{4} \rightarrow \infty}\left[\frac{1}{V_{4}}\left\langle\sum_{m} \delta^{2}\left(\lambda-\lambda_{m}\right) \sum_{n} \delta^{2}\left(\lambda^{\prime}-\lambda_{n}\right)\right\rangle_{N_{f}=2}-V_{4} \rho(\lambda) \rho\left(\lambda^{\prime}\right)\right] .
\end{aligned}
$$

The last term in (2.3) vanishes due to chiral symmetry, $\rho_{2}^{c}\left(\lambda, \lambda^{\prime}\right)=\rho_{2}^{c}\left(-\lambda, \lambda^{\prime}\right)=\rho_{2}^{c}\left(\lambda,-\lambda^{\prime}\right)=$ $\rho_{2}^{c}\left(-\lambda,-\lambda^{\prime}\right)$, and using the delta-function $\delta^{2}(z)=\lim _{\varepsilon \rightarrow 0} \varepsilon^{2} / \pi\left(|z|^{2}+\varepsilon^{2}\right)^{2}$ in the complex plane we obtain

$$
\lim _{\varepsilon \rightarrow 0} \lim _{z \rightarrow 0} \lim _{V_{4} \rightarrow \infty} \frac{1}{V_{4}} \partial_{z^{*}} \partial_{z} \log Z^{\left(N_{f}=2\right)}=\pi \rho(0) .
$$

From the effective theory of [6], with small technical modifications to allow for complex $M=\operatorname{diag}\left(z, z^{*}\right)$, we obtain for the shift in the free energy due to the BCS pairing of quarks near the Fermi surface

$$
\delta \mathscr{E}=\min _{A \in \mathrm{U}(1)}\left\{-\frac{3}{2 \pi^{2}} \Delta^{2}\left(A^{2}+A^{* 2}\right) \operatorname{det} M\right\}
$$

For $M=\operatorname{diag}\left(z, z^{*}\right)$ the minimum is obtained for $A= \pm 1$, which yields

$$
\frac{1}{V_{4}} \log Z^{\left(N_{f}=2\right)}=\frac{3}{\pi^{2}} \Delta^{2} z z^{*}+H_{2}\left(z^{2}+z^{* 2}\right)+O\left(|z|^{3}\right),
$$

where the second term on the RHS comes from a high-energy term $H_{2} \operatorname{tr} M^{2}$. (At high density the coefficient $\mathrm{H}_{2}$ can be computed perturbatively [5].) Differentiating gives

$$
\lim _{z \rightarrow 0 V_{4} \rightarrow \infty} \frac{1}{V_{4}} \partial_{z^{*}} \partial_{z} \log Z^{\left(N_{f}=2\right)}=\frac{3}{\pi^{2}} \Delta^{2},
$$

which combined with (2.6) and $d_{\text {rep }}=2$ results in (1.1). There are some technical subtleties in this derivation for which we refer to [5]. 
Let us now generalize this to an arbitrary even number of flavors. (We cannot say anything about odd $N_{f}$ since the measure is not positive definite then.) We choose the mass matrix to be $M=$ $\operatorname{diag}\left(z, \ldots, z, z^{*}, \ldots, z^{*}\right)$ with $N_{f} / 2$ entries each of $z$ and $z^{*}$. For the fundamental theory everything goes through as before, and instead of (2.6) we now obtain

$$
\lim _{\varepsilon \rightarrow 0} \lim _{z \rightarrow 0} \lim _{V_{4} \rightarrow \infty} \frac{1}{V_{4}} \partial_{z^{*}} \partial_{z} \log Z=\frac{N_{f}}{2} \pi \rho(0) .
$$

In the effective theory we obtain from the results of [6]

$$
\delta \mathscr{E}=-\frac{3 \Delta^{2}}{4 \pi^{2}} \max _{A, \Sigma_{L}, \Sigma_{R}} \operatorname{Re}\left\{A^{2} \operatorname{tr}\left(M \Sigma_{R} M^{T} \Sigma_{L}^{\dagger}\right)+A^{* 2} \operatorname{tr}\left(M \Sigma_{L} M^{T} \Sigma_{R}^{\dagger}\right)\right\}
$$

with $A \in \mathrm{U}(1)$ and $\Sigma_{L, R} \in \mathrm{SU}\left(N_{f}\right) / \mathrm{Sp}\left(N_{f}\right)$. Note that additional terms are allowed by symmetries, but their coefficients are zero in HDET. The maximum is obtained for $A= \pm 1$ and

$$
\Sigma_{L, R}= \pm\left(\begin{array}{cc}
0 & -\mathbb{1}_{N_{f} / 2} \\
\mathbb{1}_{N_{f} / 2} & 0
\end{array}\right),
$$

which gives

$$
\delta \mathscr{E}=-\frac{3 N_{f}}{2 \pi^{2}} \Delta^{2} z z^{*}
$$

Taking derivatives w.r.t. $z$ and $z^{*}$ results in (2.9) with an extra factor of $N_{f} / 2$ on the RHS. Hence we again obtain (1.1).

\subsection{QCD at nonzero isospin density}

We now consider QCD with $N_{c} \geq 2$ colors and $N_{f}=2$ flavors at nonzero isospin chemical potential $\mu_{I}=2 \mu$. The partition function of the fundamental theory is

$$
Z=\left\langle\operatorname{det}\left(D(\mu)+m_{1}\right) \operatorname{det}\left(D(-\mu)+m_{2}\right)\right\rangle_{\mathrm{YM}}=\left\langle\operatorname{det}\left(D+m_{1}\right) \operatorname{det}\left(D^{\dagger}+m_{2}\right)\right\rangle_{\mathrm{YM}}
$$

since $D(-\mu)=-D(\mu)^{\dagger}$ and the Dirac eigenvalues occur in pairs $\pm \lambda$. Choosing $M=\operatorname{diag}\left(z, z^{*}\right)$ again results in (2.6).

To construct the effective theory we note that at large $\mu_{I}$ BCS pairing of type $\left\langle\bar{d} \gamma_{5} u\right\rangle$ occurs near the Fermi surface $[9,10]$. The analysis is similar to two-color QCD, but the coset space is now $\mathrm{U}(1)_{A} \times \mathrm{U}(1)_{I_{3}}$ [7], where the latter is the $\mathrm{U}(1)$ symmetry with respect to the third isospin generator. For the free-energy shift we obtain

$$
\delta \mathscr{E}=-c_{\text {iso }} \Delta^{2} \max _{A \in \mathrm{U}(1)}\left\{\left(A^{2}+A^{* 2}\right) \operatorname{det} M\right\}=-2 c_{\text {iso }} \Delta^{2} z z^{*}
$$

with $A= \pm 1$ in the last step. $c_{\text {iso }}$ is a positive coefficient that can be computed in HDET following $[11,6]$. Using a result of [12] we obtain $c_{\text {iso }}=3 N_{c} / 4 \pi^{2}$, which gives

$$
\frac{1}{V_{4}} \log Z\left(z, z^{*}\right)=\frac{3 N_{c}}{2 \pi^{2}} \Delta^{2} z z^{*}+H_{2}\left(z^{2}+z^{* 2}\right)+O\left(|z|^{3}\right)
$$

and thus (1.1) with $d_{\text {rep }}=N_{c}$. 


\subsection{Adjoint QCD}

We now to turn to QCD with fermions transforming in the adjoint representation of $\mathrm{SU}\left(N_{c}\right)$. We again consider an even number of flavors ${ }^{1}$ and $M=\operatorname{diag}\left(z, \ldots, z, z^{*}, \ldots, z^{*}\right)$. The partition function of the fundamental theory is

$$
Z=\left\langle\operatorname{det}^{N_{f} / 2}(D+z) \operatorname{det}^{N_{f} / 2}\left(D+z^{*}\right)\right\rangle_{\mathrm{YM}}=\left\langle\operatorname{det}^{N_{f} / 2}(D+z) \operatorname{det}^{N_{f} / 2}\left(D^{\dagger}+z^{*}\right)\right\rangle_{\mathrm{YM}},
$$

where in the last step we have used the anti-unitary symmetry $\left[C \gamma_{5} K, D\right]=0$ of the Dirac operator with adjoint fermions. In the fundamental theory we thus obtain (2.10) again.

The construction of the low-energy effective theory for this case proceeds in analogy to [6], except that the coset space is now $\mathrm{U}(1)_{B} \times \mathrm{U}(1)_{A} \times\left[\mathrm{SU}\left(N_{f}\right)_{L} / \mathrm{SO}\left(N_{f}\right)_{L}\right] \times\left[\mathrm{SU}\left(N_{f}\right)_{R} / \mathrm{SO}\left(N_{f}\right)_{R}\right]$. For the free-energy shift we obtain with $A \in \mathrm{U}(1)$ and $\Sigma_{L, R} \in \mathrm{SU}\left(N_{f}\right) / \mathrm{SO}\left(N_{f}\right)$

$$
\begin{aligned}
\delta \mathscr{E} & =-c_{\mathrm{adj}} \Delta^{2} \max _{A, \Sigma_{L}, \Sigma_{R}} \operatorname{Re}\left\{A^{2} \operatorname{tr}\left(M \Sigma_{R} M^{T} \Sigma_{L}^{\dagger}\right)+A^{* 2} \operatorname{tr}\left(M \Sigma_{L} M^{T} \Sigma_{R}^{\dagger}\right)\right\} \\
& =-c_{\mathrm{adj}} \Delta^{2} \cdot 2 N_{f} z z^{*} \quad \text { for } \quad A= \pm 1, \quad \Sigma_{L, R}= \pm\left(\begin{array}{cc}
0 & \mathbb{1}_{N_{f} / 2} \\
\mathbb{1}_{N_{f} / 2} & 0
\end{array}\right) .
\end{aligned}
$$

Again, additional terms are allowed by symmetries, but their coefficients are zero in HDET. To compute the coefficient $c_{\text {adj }}$ in HDET we note that diquark condensation again occurs in the pseudoscalar channel [7] but that the diquark condensate is now symmetric in the color and flavor indices (and antisymmetric in the spin indices as before). Choosing the phases of the right- and left-handed condensates so as to minimize the ground-state energy we obtain after some calculations $c_{\text {adj }}=3\left(N_{c}^{2}-1\right) / 8 \pi^{2}$ and thus ${ }^{2}$

$$
\frac{1}{V_{4}} \log Z\left(z, z^{*}\right)=\frac{3\left(N_{c}^{2}-1\right)}{4 \pi^{2}} N_{f} \Delta^{2} z z^{*}+H_{2} \frac{N_{f}}{2}\left(z^{2}+z^{* 2}\right)+O\left(|z|^{3}\right) .
$$

Taking derivatives w.r.t. $z$ and $z^{*}$ again results in (1.1), now with $d_{\text {rep }}=N_{c}^{2}-1$.

\section{Consistency with microscopic limit and random matrix theory}

Let us define the spectral density in a finite volume in analogy to (2.4),

$$
\bar{\rho}(\lambda)=\lim _{z \rightarrow 0} \frac{1}{V_{4}}\left\langle\sum_{n} \delta^{2}\left(\lambda-\lambda_{n}\right)\right\rangle_{N_{f}},
$$

where $z \rightarrow 0$ indicates that we only consider the chiral limit. To resolve individual eigenvalues near zero one defines the so-called microscopic spectral density $\rho_{s}$ by rescaling the eigenvalues with an appropriate power of the four-volume, which in our case is $\sqrt{V_{4}}$. It is well known in many

\footnotetext{
${ }^{1} N_{f}$ must be even if we want to use a mass term of the form $M=\operatorname{diag}\left(z, \ldots, z, z^{*}, \ldots, z^{*}\right)$. With real masses we could also consider odd $N_{f}$ since for adjoint fermions the measure is positive definite in this case. However, then the $\Delta^{2}$ and $H_{2}$ terms in (2.18) have the same mass dependence, i.e., they are proportional to $m_{1}^{2}+m_{2}^{2}+\ldots$ This is because for real masses the maximum in (2.17) is obtained for $\Sigma_{L, R}= \pm \mathbb{1}_{N_{f}}$. For two-color QCD this argument is not applicable since $\pm \mathbb{1}_{N_{f}}$ is not in the coset space $\mathrm{SU}\left(N_{f}\right) / \mathrm{Sp}\left(N_{f}\right)$. E.g., for $N_{f}=2$ with real masses we have $\delta \mathscr{E} \propto m_{1} m_{2}$, see (2.7).

${ }^{2}$ Note that the factor of $N_{f} / 2$ in the $H_{2}$ term was erroneously omitted in [5, eq. (60)].
} 
other cases that $\rho_{s}$ is a universal function that only depends on the symmetries and the symmetrybreaking pattern of the system. Therefore it can very economically be computed in the simplest theory respecting these symmetries, which is random matrix theory (RMT). RMT can also be understood as a zero-dimensional limit of the effective theory. To match results from the physical theory with RMT results a physical scale has to be set. This can be done, e.g., by matching the mass-dependence of the partition functions of the effective theory and of RMT. In the following we show, for all three cases we considered, that the correct rescaling is given by

$$
\rho_{s}(\xi)=\lim _{V_{4} \rightarrow \infty} \frac{2 \pi^{2}}{3 d_{\mathrm{rep}} \Delta^{2}} \bar{\rho}\left(\sqrt{\frac{2 \pi^{2}}{3 d_{\mathrm{rep}} V_{4} \Delta^{2}}} \xi\right)
$$

and that the asymptotic behavior of the RMT result for $\rho_{s}(\xi)$ is consistent with (1.1).

Let us begin with two-color QCD. In this case (3.2) with $d_{\text {rep }}=2$ was already proposed in [6]. The corresponding RMT was constructed in [13], and the analytical RMT result for $\rho_{s}(\xi)$ was computed in [14]. From this result one finds

$$
\lim _{|\xi| \rightarrow \infty} \rho_{s}(\xi)=\frac{1}{\pi}
$$

Note that (3.2) is also valid for $|\xi| \rightarrow \infty$ but that on the RHS the thermodynamic limit is taken first. Hence (3.2) and (3.3) imply

$$
\frac{2 \pi^{2}}{3 d_{\mathrm{rep}} \Delta^{2}} \bar{\rho}(0)=\frac{1}{\pi}
$$

which agrees with (1.1). Note that this should not be considered as a derivation of (1.1) but as a consistency check.

For QCD with isospin chemical potential the mapping of the physical theory to RMT was derived in [7, Sec. 4.2], except that a prefactor still needed to be computed that can be fixed by matching (2.15) and [7, Eq. (4.37)]. Using this prefactor in [7, Eq. (4.49)] then gives (3.2) with $d_{\text {rep }}=N_{c}$. The analytical RMT result for $\rho_{s}(\xi)$ has been computed previously [7, Eq. (4.51)]. Adapting this result to our case shows that (3.3) holds so that we again obtain the consistency check (3.4).

For adjoint QCD the mapping of the physical theory to RMT was derived in [7, Sec. 4.3], again with the exception of a numerical prefactor. The microscopic spectral density for this case can be obtained as a certain limit of a more general result [15]. The missing prefactor is obtained by matching (2.18) for $N_{f}=2$ with [15, Eq. (4.17)]. Using this prefactor in [15, Eq. (4.9)] again gives (3.2), now with $d_{\text {rep }}=N_{c}^{2}-1$. From the analytical RMT result for $\rho_{s}(\xi)$ (modulo a factor of 4 due to different conventions) we can again conclude that (3.3) and thus also (3.4) holds.

\section{Conclusions}

We have shown that three QCD-like theories at high density obey a Banks-Casher-type relation that relates the BCS gap to the two-dimensional Dirac density at the origin. The relation is formally identical for all three cases and only differs in the dimension of the gauge group representation in 
which the fermions transform. To derive the Banks-Casher-type relation we also constructed the hitherto unknown low-energy effective theories for two of the cases. In the derivation of the BanksCasher-type relation it is essential that the fermionic measure is positive definite. Therefore we cannot generalize our result to the color-superconducting state of QCD at high density. Presumably the connection between the Dirac spectrum and the BCS gap is more complicated in this case, similar to the situation at low density [3, 4].

Our results, together with other known results for microscopic spectral correlations and spectral sum rules, can in principle be checked in lattice simulations since there is no sign problem. However, it is difficult to reach densities high enough for our analysis to be valid and still keep the lattice spacing reasonably small.

\section{References}

[1] T. Banks and A. Casher, Chiral symmetry breaking in confining theories, Nucl. Phys. B169 (1980) 103.

[2] H. Leutwyler and A. V. Smilga, Spectrum of Dirac operator and role of winding number in QCD, Phys. Rev. D46 (1992) 5607.

[3] J. C. Osborn, K. Splittorff, and J. J. M. Verbaarschot, Chiral symmetry breaking and the Dirac spectrum at nonzero chemical potential, Phys. Rev. Lett. 94 (2005) 202001 [hep-th/ 0501210 ].

[4] J. C. Osborn, K. Splittorff, and J. J. M. Verbaarschot, Chiral Condensate at Nonzero Chemical Potential in the Microscopic Limit of QCD, Phys. Rev. D78 (2008) 065029 [arXiv: 0805.1303 ].

[5] T. Kanazawa, T. Wettig, and N. Yamamoto, Banks-Casher-type relation for the BCS gap at high density, Eur.Phys.J. A49 (2013) 88 [arXiv:1211.5332].

[6] T. Kanazawa, T. Wettig, and N. Yamamoto, Chiral Lagrangian and spectral sum rules for dense two-color QCD, JHEP 08 (2009) 003 [arXiv: 0906.3579 ].

[7] T. Kanazawa, Dirac Spectra in Dense QCD. Springer theses. Springer Japan, 2013.

[8] D. K. Hong, An effective field theory of QCD at high density, Phys. Lett. B473 (2000) 118 [hep-ph/9812510].

[9] D. T. Son and M. A. Stephanov, QCD at finite isospin density, Phys. Rev. Lett. 86 (2001) 592 [hep-ph/0005225].

[10] D. T. Son and M. A. Stephanov, QCD at finite isospin density: From pion to quark antiquark condensation, Phys. Atom. Nucl. 64 (2001) 834 [hep-ph/0011365].

[11] T. Schafer, Mass terms in effective theories of high density quark matter, Phys. Rev. D65 (2002) 074006 [hep-ph/0109052].

[12] M. Hanada and N. Yamamoto, Universality of Phases in QCD and QCD-like Theories, JHEP 02 (2012) 138 [arXiv:1103.5480].

[13] T. Kanazawa, T. Wettig, and N. Yamamoto, Chiral random matrix theory for two-color QCD at high density, Phys. Rev. D81 (2010) 081701 [arXiv:0912.4999].

[14] G. Akemann, T. Kanazawa, M. J. Phillips, and T. Wettig, Random matrix theory of unquenched two-colour QCD with nonzero chemical potential, JHEP 03 (2011) 066 [arXiv: 1012.4461 ].

[15] G. Akemann, The complex Laguerre symplectic ensemble of non-Hermitian matrices, Nucl. Phys. B730 (2005) 253 [hep-th/ 0507156$].$ 